ORION, Vol. 10, No. 1/2, pp. 1-32

ISSN 0259-191-X

\title{
A CHOICE REDUCTION MODEL FOR THE CONCEPT PHASE OF A WEAPON SYSTEM
}

\author{
C. M. ERASMUS \\ Dynamics Design Group \\ Kentron \\ South Africa
}

\begin{abstract}
During the concept phase of a weapon system it is often necessary to decide between a number of different development or purchasing options. This typically involves using a multidimensional value system that incorporates aspects such as performance, cost, risk, logistics and time-scales.

This paper describes a model which assists in the choice in the performance dimension by including application battlefield scenarios in the analysis, and calculating the relative worth of competing systems based on the expected utility of the component weapon system attributes.

The author would like to acknowledge the contributions of Mr BL Logan and Mr AE Heijdenrijch in the development of the model. This project would not have been possible without their suggestions and support, especially on the detail procedures for implementing the model.
\end{abstract}

\section{ABBREVIATTIONS}

AHP - $\quad$ Analytic Hierarchy Process

ATM - $\quad$ Anti-tank (Guided) Missile

CIM - Cross-impact Matrix

E - Expected Value

$E[X]$ - Expected Value of the variable $X$

II - Image Intensifier

MOE - Measure of Effectiveness 


$\begin{array}{ll}\text { ROC - } & \text { Required Operational Capability } \\ \text { SC - } & \text { Scenario } \\ \text { SCA - } & \text { Scenario Attribute } \\ \text { Tel - } & \text { Telescope } \\ \text { TI - } & \text { Thermal Imager } \\ \text { WS - } & \text { Weapon System } \\ \text { WSA - } & \text { Weapon System Attribute }\end{array}$

\section{SCOPE AND STRUCTURE}

The structure of the body of the paper is as follows:

Section 1 serves as introduction by describing the problem, the value system, and the user-specified functional criteria forming the design drivers in the process.

Section 2 describes the implementation problems experienced using the classical and AHP approaches.

Section 3 discusses the changes to the model to accommodate the problems, under the following headings:

- Conceptual model;

- WSAs: Designer options;

- Scenario attributes and distributions;

- Interaction between WSAs and SCAs;

- Utility functions;

- Modelling CIM interactions; and

- Specification of utility functions.

Section 4 discusses the implementation of the CIM model, under the following headings:

- Generation of scenarios and SCA distributions;

- Incorporating different development options; and

- Incorporating different scenarios.

Section 5 concludes the paper with a summary of the procedure for constructing 
the cross-impact matrix, and an overview of the current status of the model.

Appendix A presents closed-form expressions for the expected use of a weapon system attribute, given a triangular distribution for the scenario attribute(s), and a specific class of utility functions. It also presents formulas for the calculation of the parameters of a triangular distribution, given estimates of two percentiles and either the mean or the mode.

\section{INTRODUCTION}

\section{Problem description}

During the concept phase in the life cycle of a new weapon system a number of concepts that could fulfil the intended mission of the WS are generated by the system team. There may be attributes such as range, sighting system, accuracy, etc. that vary widely between the different weapon system options. There are usually so many of these factors, and so many interdependencies between them, that it tends to be difficult to choose between these options. In addition, the decisions typically have to be made relative to more than one dimension, such as - Performance

- Cost

- Technical feasibility/risk

- Time-scales

A choice reduction model assists in reducing the number of concepts in an orderly fashion, using trade-offs between the different dimensions. As the project progresses through the concept phase, one usualiy finds that the detail of modelling increases as the number of concepts decreases, as shown schematically in Figure 1.

\section{Value system}

Looking at the joint dimensions of Performance, Cost, and Risk, the basic idea behind the model is to calculate a normalized score for each concept with respect to each dimension, as indicated in Table 1. 


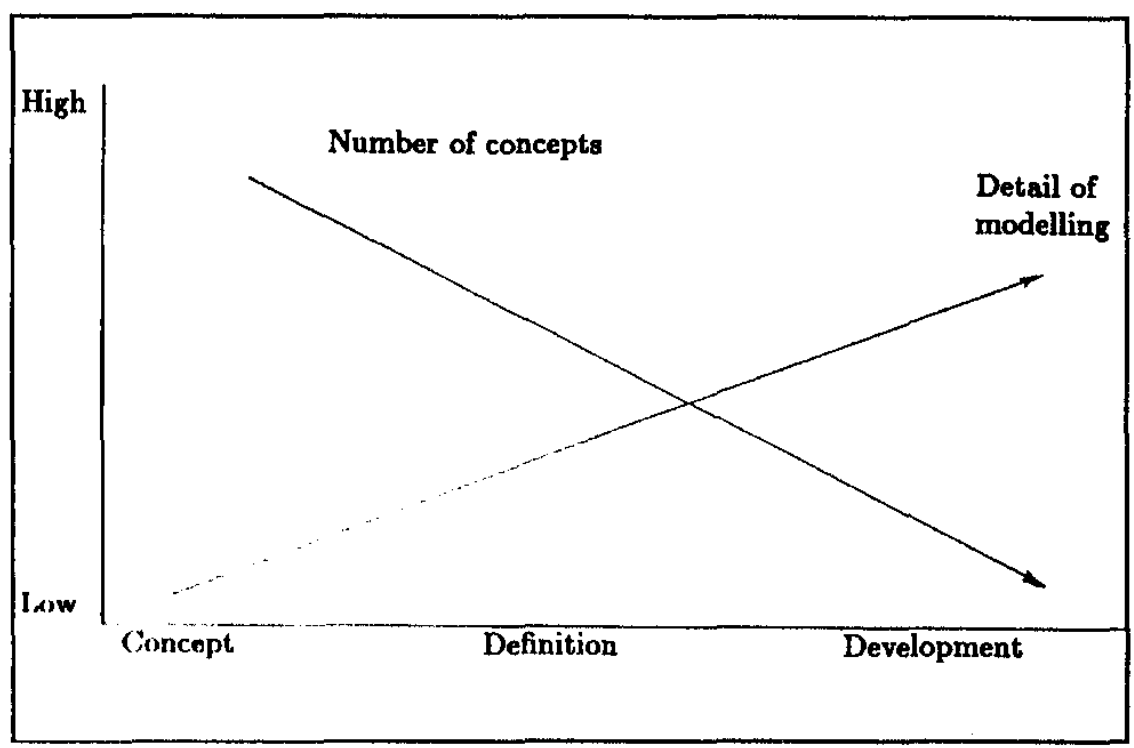

Figure 1: Number of concepts vs Detail of modelling

\begin{tabular}{|c|c|c|c|}
\hline & Performance & Technical Risk & Cost \\
\hline Concept 1 & $p_{1}$ & $r_{1}$ & $c_{1}$ \\
Concept 2 & $p_{2}$ & $r_{2}$ & $c_{2}$ \\
$\cdot$ & $\cdot$ & $\cdot$ & $\cdot$ \\
$\cdot$ & $\cdot$ & $\cdot$ & $\cdot$ \\
Concept $n$ & $p_{\mathrm{n}}$ & $r_{\mathrm{n}}$ & $c_{\mathrm{n}}$ \\
\hline & 100 & 100 & 100 \\
\hline
\end{tabular}

Table 1: Normalised scores

The choice reduction process then involves determining a cut-off point with respect to each dimension, resulting in each concept being allocated to one of eight hyperquadrants, as indicated in Figure 2. Those concepts falling in the hashed hyperquadrant are carried over to the next round of more detailed modelling.

In this paper we describe the development of the model in the performance dimension, with the corresponding development in the other two dimensions currently in progress. The performance submodel uses battlefield scenarios to calculate the relative worth of competing systems based on the expected utility of the component weapon system attributes. The methodology will be explained 
using an ATM for the infantry as an example.

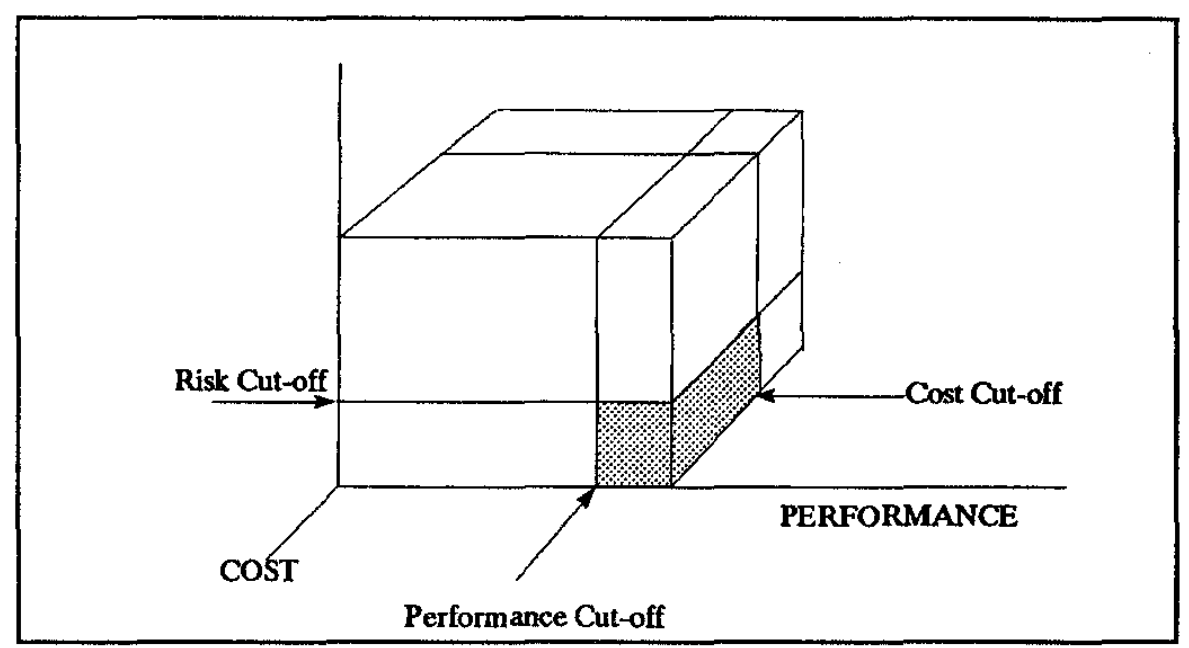

Figure 2: Choice reduction process

\section{Design criteria}

The high-level functional requirements for a WS are normally specified by the User Requirement (ROC) document. For the Infantry ATM example the criteria could read as follows:

- $\quad$ More than one missile per man, maximum two men/system;

- Portable (40 kg between two men);

- $\quad$ Ability to track a $30 \mathrm{~km} / \mathrm{h}$ crossing speed target;

- $\quad$ Arming further than $50 \mathrm{~m}$ away;

- $\quad$ Maximum range at least $2 \mathrm{~km}$;

- $\quad$ Flight time to maximum range at most $13 \mathrm{~s}$;

- Terminal effect must stop a T72 tank;

- Night-fighting capability;

- $\quad$ Ease of use;

- Hit probability of $95 \%$ against a standard NATO target over the whole range;

- Air-drop capability; and

- $\quad$ Flexibility of firing height.

These criteria could be overlapping, some may be superfluous, irrelevant, and even contradictory. They are also not of equal importance as design drivers for the WS. The choice reduction process attempts to quantify these aspects and to incorporate them into the model. 


\section{MODEL DEVELOPMENT}

\section{The classical formulation}

Initially it was decided to use the standard pair-wise comparison technique to rank the design criteria with respect to one another, resulting in a normalized set of weights for the criteria. Having established these weights, each concept would be scored in terms of how well it performed with respect to each of the criteria, and the weighted sum of these scores would yield an overall performance score for the concopt.

A pair-wise comparison table for the ATM example design criteria is given in Table 2. The selected user group is presented with a list of definitions for the criteria. They are then asked to complete the table where, for example, a code $A 2$ in the $A$ vs $X$ comparison means that criterion $A$ is strongly more important than criterion $X$, whereas $X 2$ indicates the converse, with 0 indicating indifference. A set of weights is then assigned to the 5 possible codes, and the weighted total for each criterion is calculated, and normalized over the criteria.

The choice of weights assigned to the codes presents a problem, as there are different ways in which they can justifiably be chosen, which can clearly lead to different rankings between the criteria. In the literature this choice has also been done in different ways. Aspects that should be considered are:

How are questionnaires to be combined over respondents?

- If the individual scores are combined arithmetically, should the $X 2$ and $X 1$ weights not be taken as 0 to eliminate double counting?

- Is the underlying preference scale an arithmetic or geometric one? In the latter case the $X$ code weight should be the inverse of the corresponding $A$ weight. In the former, the no-preference weight should be set to 0 so as not to be included in the total. 


\begin{tabular}{|c|c|c|c|c|c|c|c|c|c|c|c|c|c|c|c|c|}
\hline \multirow[b]{2}{*}{ REQUIREMENTS } & \multirow[b]{2}{*}{$\begin{array}{l}\text { REF. NR } \\
\text { OF DEF. }\end{array}$} & \multirow[b]{2}{*}{ CODE } & \multicolumn{14}{|c|}{ REQUIREMENTS } \\
\hline & & & A & B & $C$ & $\mathrm{D}$ & $E$ & $\mathrm{~F}$ & G & $\mathrm{H}$ & 1 & $J$ & K & L & M & $\mathrm{N}$ \\
\hline Man-portable & 2.1 & A & & $\mathrm{A} 2$ & A1 & 0 & A2 & 0 & G1 & $\mathrm{A} 2$ & 12 & $\mathrm{~A} 2$ & $\mathrm{~A} 2$ & $\mathrm{~L} 1$ & M1 & A1 \\
\hline Maximum range & 2.2 & B & - & & $\mathrm{B} 1$ & B1 & B1 & $\mathrm{B} 2$ & G1 & $\mathrm{B} 1$ & 11 & B1 & $\mathrm{B} 2$ & B1 & M1 & B2 \\
\hline Minimum safe distance & 2.3 & $\mathrm{C}$ & - & - & & D1 & E2 & $\mathrm{C} 1$ & G1 & 0 & 12 & 0 & $\mathrm{C} 2$ & $\mathrm{C} 1$ & M2 & C1 \\
\hline Flight time & 2.4 & $D$ & - & - & - & & E1 & D1 & G1 & D2 & 11 & D1 & $\mathrm{D} 2$ & D2 & M1 & D1 \\
\hline Terminal effect & 2.5 & $E$ & - & - & - & - & & E2 & E1 & E1 & 0 & E1 & E2 & $\mathrm{E} 2$ & E1 & E2 \\
\hline Night-fighting capability & 2.6 & $\mathrm{~F}$ & - & - & - & - & - & & $\mathrm{G} 1$ & $\mathrm{H} 1$ & 12 & $\mathrm{~J} 2$ & $\mathrm{~F} 1$ & $\mathrm{~L} 1$ & M2 & N1 \\
\hline Operator safety & 2.7 & $\mathbf{G}$ & - & - & - & - & - & - & & G1 & 12 & $\mathrm{~J} 1$ & G1 & G1 & M2 & G1 \\
\hline Ease of use & 2.8 & $\mathrm{H}$ & - & - & - & - & - & - & - & & 11 & $\mathrm{J1}$ & $\mathrm{H} 2$ & $\mathrm{H} 1$ & M1 & N1 \\
\hline Accuracy (Maximum) & 2.9 & 1 & - & - & - & - & - & - & - & - & & 12 & 12 & 11 & 0 & 11 \\
\hline Accuracy (Minimum) & 2.10 & $J$ & - & - & - & - & - & - & - & - & - & & $\mathrm{K} 2$ & $\mathrm{~L} 1$ & 0 & N2 \\
\hline Air-drop capability & 2.11 & $\mathrm{~K}$ & - & - & - & - & - & $=$ & - & - & - & - & & L1 & M2 & 0 \\
\hline Multiplatform capability & 2.12 & $\mathrm{~L}$ & - & - & - & - & - & - & - & - & - & - & - & & M2 & L1 \\
\hline Counter-measure resistance & 2.13 & $M$ & - & - & - & - & - & - & - & - & - & - & - & - & & M2 \\
\hline Flexibility of firing height & 2.14 & $\mathrm{~N}$ & - & - & - & - & - & - & - & - & - & - & - & - & - & \\
\hline
\end{tabular}

Table 2: Pair-wise comparison table 
Having calculated the individual questionnaire weights and combined them over the respondents, it now remained to score each of the concepts in terms of the extent to which it achieves each of the criteria. This would be on a scale of 0 to 1 , with 0 signifying no achievement, and 1 total achievement. To derive these achievement scores would likely require a fair amount of low-level analysis and simulation that would be highly dependent on the specific WS under consideration. Having obtained these, the final concept score can again be calculated as a weighted average of the achievement scores, using the criteria weights.

\begin{tabular}{|l|l|l|}
\hline Criterion & Paratroopers & Infantry \\
\hline $\mathrm{A}=$ Man-portable & 0,024 & 0,059 \\
\hline $\mathrm{B}=$ Maximum Range & 0,097 & 0,055 \\
\hline $\mathrm{C}=$ Minimum Safe Distance & 0,024 & 0,021 \\
\hline $\mathrm{D}=$ Flight Time & 0,053 & 0,042 \\
\hline $\mathrm{E}=$ Terminal Effect & $0,151(1)$ & $0,182(1)$ \\
\hline $\mathrm{F}=$ Night-fighting Capability & 0,097 & 0,086 \\
\hline $\mathrm{G}=$ Operator Safety & 0,023 & 0,035 \\
\hline $\mathrm{H}=$ Ease of Use & 0,022 & 0,030 \\
\hline $\mathrm{I}=$ Accuracy (Maximum) & $0,140(2)$ & $0,153(2)$ \\
\hline $\mathrm{J}=$ Accuracy (Minimum) & 0,092 & 0,023 \\
\hline $\mathrm{K}=$ Air-drop Capability & $0,103(3)$ & $0,039(10)$ \\
\hline $\mathrm{L}=$ Multiplatform Capability & 0,037 & 0,084 \\
\hline $\mathrm{M}=$ Counter-measure Resistance & 0,102 & $0,098(3)$ \\
\hline $\mathrm{N}=$ Flexibility of Firing Height & 0,035 & 0,094 \\
\hline INCONSISTENCY: & 0,162 & 0,226 \\
\hline
\end{tabular}

Table 3: Ranking per User Group

We completed a trial analysis using the classical approach, but were not comfortable with the results, specifically as far as the non-uniqueness of the choice of code weights is concerned. 


\section{The AHP formulation}

We decided to use the Analytical Hierarchy Process as developed by Saaty (Ref [1] and [5]) to establish the criteria weights. The AHP has been designed for decision problems that can be structured as a hierarchy. The basic idea is that a number of alternatives or actions exist which contribute to the attainment of some overall goal through a hierarchy of criteria, which may have many levels.

To demonstrate the application of the model using the AHP weight, the pair-wise comparison matrix for the ATM design criteria was constructed by two user groups. These were members of the infantry versus a group of paratroopers. To cast our problem in the AHP mould, we took our user groups to be on the first level below the goal (i.e. they correspond to the AHP criteria), whilst our criteria correspond to the alternatives on the lowest level of the AHP hierarchy.

The resultant set of weights given in Table 3 are thus local priorities in the AHP formulation. To obtain the global priorities, the user groups have to be compared with respect to one another. Apart from the (mostly political, but very real) problems encountered in attempting to do this, other patterns and problems to be noted in the table are:

- Terminal Effect and Accuracy at maximum range rank as most important for both user groups.

- $\quad$ Air-drop capability ranks third for the Paratroopers, but tenth for the Infantry.

- There is a relatively small spread ( $2 \%$ to $16 \%)$ between the weights, without a marked Pareto effect. None of the criteria stand out as being either markedly inferior or superior to the others.

The inconsistency level of the Infantry user group is uncomfortably high. The weight depend on the user group. From user feedback it was also clear that the comparative scores were highly influenced by assumed application scenarios for the WS.

During the analysis the difference in importance attached to design criteria by different user groups stood out clearly. It also became apparent that the envisaged application environment of the weapon system would have to be quantified more 
explicitly to obtain the necessary resolution in the value system to be able to prioritize the different criteria. An example is that accuracy at minimum range would be more important than accuracy at maximum range if the engagement took place in bush at close contact, whilst the converse would be true if the engagement took place in an open area. We came to the conclusion that the Classical/AHP approach failed to provide a satisfactory choice reduction mechanism in the performance dimension of the model in Table 1.

The next section describes a way in which the application scenario can be incorporated in the analysis.

\section{THE CROSS-IMPACT MATRIX}

\section{Conceptual model}

It is apparent that the interactions between the proposed or possible WS attributes and the possibie application scenarios of the WS need to be quantified explicitly. This can be done by way of a cross-impact matrix with the WS attributes across the top, and the scenario attributes down the side, as shown schematically in Table 4. Before discussing the structure of the matrix, some comments on the use of scenario analysis to quantify design options are in order. The basic principle is simple: Given the wisdom of foresight, one would be able to design a weapon system that would cope exactly with all the situations in which it would be applied, without any unnecessary or underutilized capabilities.' In the absence of perfect knowledge, the best that can be done is to rely on the judgement of experts in various applicable fields, and to define various scenarios in which the WS could be employed. Depending on the scenario, some features of the WS will be utilized more than others. The expected use of the features can then be traded off in a cost-benefit analysis, to assist in the decision as to which features should be included in the WS. The use of scenario analysis as a decision support tool is well established. Reference [2] discusses its use in planning the operational test and evaluation of systems. Closer to our requirements, Reference [3] Chapter 42 , illustrates the use of scenarios in performing a comparative weapon system analysis between tanks and ATMs. The (futuristic) scenario described there is similar to what we need for our analysis. Given the scenario, they use (proven) 
simulation models to estimate the kill rates between the various weapon systems, and use these to translate the fire-power of the different weapon systems to a comparable MOE. In our case, the comparison will be done on a weighted combination of the expected utilization of the different WS features.

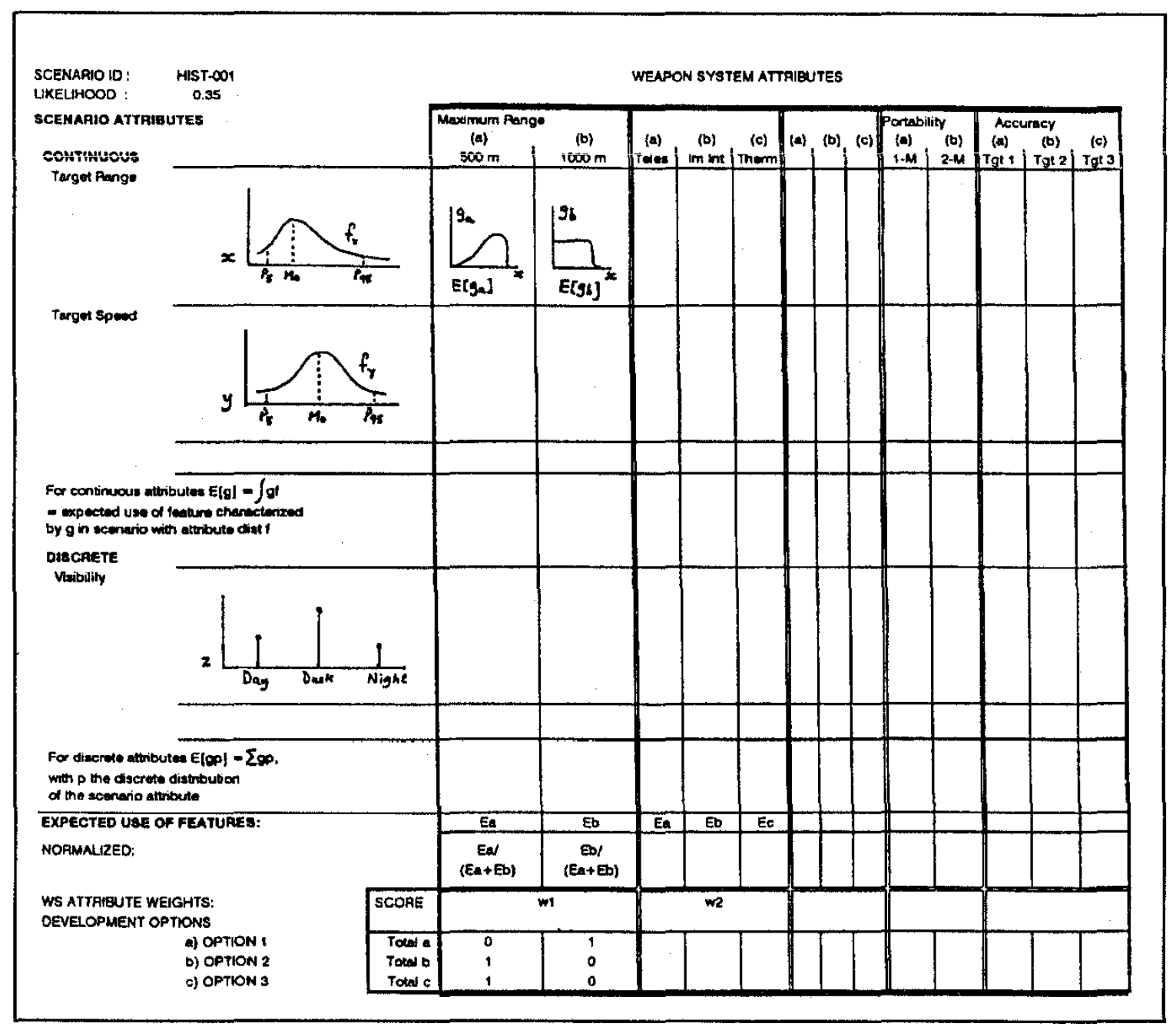

Table 4: Conceptual model

\section{Weapon system attributes}

A missile system, seem as a functional entity, has a number of attributes/capabilities such as range, night fighting, terminal effectiveness, accuracy, reliability, mass, and so on. The problem facing the designer is that he has a number of different options/features for each attribute, for example, he can choose to fit the missile with ordinary telescopic sight only, or with an image intensifier in addition, or with thermal imaging as well. The latter option will 
provide the system with a full 24 hour fighting capability, but obviously at much increased cost. What the model attempts to do is to calculate the expected benefit or use of the different design options within each attribute, with respect to the proposed scenarios in which the WS will be employed. It is important that the designer has clarity over which attributes he wants to include in the evaluation list, as well as the options available within each. The scale on which the options are measured must also be clearly established. For a discrete attribute such as the sight example mentioned above, the categories are discrete classes, whilst for a continuous attribute such as range or flight time, the cut-offs on the design scale must be determined.

\section{Scenario attributes and distributions}

For each WSA, specific information about the application scenario will be needed in order to quantify the extent to which the options within that attribute will be utilized during the application. For example, one would have to know what portion of the engagement takes place in total darkness to determine the extent to which the night-sight capability is utilized. The corresponding scenario attribute (SCA) that will thus have to be known is that of Visibility. As with the WSA, the scale on which the SCA is measured must be clearly established - it can also be discrete or continuous. It would be prudent to choose the SCA scale to map directly onto the corresponding WSA scale in the case of a one-to-one situation, if possible. This simplifies matters somewhat as it makes for one le'ss translation step. For the 'Sight' WSA, the SCA attribute 'Visibility' may be measured on the scale 'Day, Dusk, Night', for example.

The SCA distributions must be quantified in some way so as to be able to calculate the expected usage of the WSAs. This would be done using a distribution which is both realistic, and easy to manipulate from an analytical/simulation point of view. The Gaussian distribution, in spite of a very strong theoretical foundation, has the disadvantage in practice that is perfectly symmetrical, and allows impossibly low or high values. An SCA like target range, for instance, is definitely skew, with specific upper and lower limits. A transformation such as the two- or threeparameter Log-Normal can be used, but this has other drawbacks. A distribution 
that is a fair compromise between the conflicting requirements mentioned above is the Triangular distribution, which is completely determined by specifying its minimum value, the mode (most likely value), and the maximum value. For our application, it is important that the parameters for the distributions van easily be obtained by a structured interview session with a group of users/experts. Appendix A presents the formulas to calculate the parameters of a triangular distribution, given estimates of, say, the $5^{\text {th }}$ and $95^{\text {th }}$ percentiles, and the mode or the mean.

To summarize in statistical terms, an SCA is measured in terms of the variable $X$, and the distribution of $X$ is characterized by a probability density function $f_{X}(X)$.

\section{Interaction between WSAs and SCAs}

It may be that more than one SCA influences the usage of one WSA. An example of this would be that both the target range and target speed determine the sightline rotation rate to which the missile is subjected. Conversely, it can also happen that one SCA influences more than one WSA, for example the target speed influences both the sight-line rotation rate as well as the missile accuracy. The ideal would be to have a 'pure' dependence between WSAs and SCAs, in the sense that the WSAs do not influence one another. This is unfortunately not always the case. Missile accuracy, for example, is determined not only by target speed, size, and crossing distance, but also by the missile speed itself. The missile mass is another WSA that interacts with many other WSAs. For the one-on-one situation, these dependencies can be quantified directly via the utility functions described in the next paragraph.

\section{Utility functions}

Given a set of SWAs and the corresponding SCAs that have an impact on them, the model attempts to quantify the interactions between them via a set of utility functions. The range of a utility function varies between 0 and 1 , and is defined on the same axis as the corresponding SCA. It measures the extent to which the WSA option is used as a function of the level of the SCA. For example, if one of the warhead options was 'Penetration ability (RHA) $=800 \mathrm{~mm}$ ' and a target with 
an equivalent RHA value of $200 \mathrm{~mm}$ was encountered, then only 0,25 of this feature would be utilized. Conversely, if the target RHA value was indeed 800 $\mathrm{mm}$, then the feature would be utilized at a level of 1 . In the extreme, for a target with an RHA value greater than $800 \mathrm{~mm}$, and which the warhead fails to put out of action, one could argue that the utilization drops to zero again.

In practice, one could thus have a utility function that starts from zero, and increases from a certain point where the WS feature becomes useful, up to a maximum value of 1 where the WS is utilized at its maximum, after which it decreases to zero again. Both the increase and/or the decrease could be steps.

As an alternative, one could have a utility function that starts at 1 , and after a certain point drops to zero. A maximum range capability of, say, $1500 \mathrm{~m}$ is a possible example of this - for all targets up to a range of $1500 \mathrm{~m}$ one could argue that the capability is fully utilized, whereas for targets beyond this range the capability becomes irrelevant. Other shapes of utility functions are also possible. The two mentioned above are sketched in Figures 3 and 4 respectively.

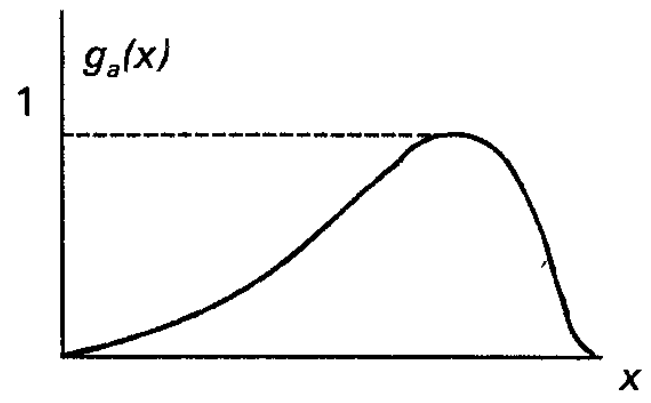

Figure 3: Utility of feature $a$, as a function $g_{a}(x)$ of SCA level $x$.

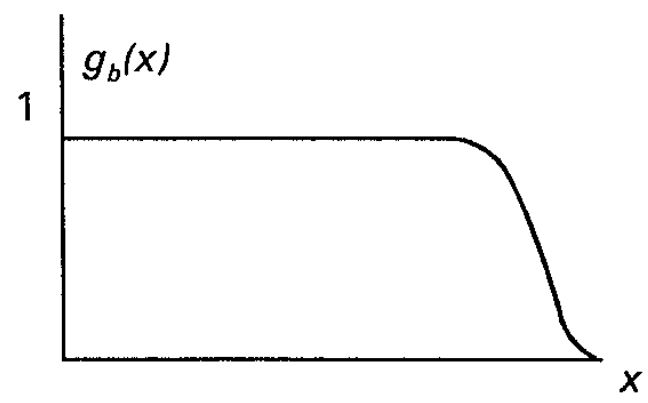

Figure 4: Utility of feature $b$, as a function $g_{b}(x)$ of SCA level $x$. 
Summarizing the interpretation of $g_{a}(x)$ and $E\left[g_{a}\right]$ in mathematical terms:

The interpretation of $g_{a}(x)$ is: If the SCA was on level $x$, then $g_{a}(x)$ of feature ' $a$ ' would have been utilized, if it was available.

The expected use of a feature (see Appendix $A$ ) is synonymous with the expected values of $g(X)$, i.e.

$$
E_{a}=E\left[g_{a}(X)\right]=\int g_{a}(x) f_{x}(x) d x
$$

The interpretation of $E_{a}$ (the expected use of feature ' $a$ ') is:

Given the application scenario, feature ' $a$ ' would (assuming it was available) be utilized (on the average) on a level $E_{a}$, between 0 and 1 .

If the WSA under consideration only has the two options/features ' $a$ ' and ' $b$ ', the normalized use (refer to Table 4) would be calculated as:

$$
e_{a}=E_{a} /\left(E_{a}+E_{b}\right) ; \quad e_{b}=E_{b} /\left(E_{a}+E_{b}\right)
$$

\section{Modelling CIM interactions}

The above discussion has been for the one-on-one situation with one SCA affecting one WSA. If more than one SCA affects the same WSA, for example, in addition to SCA (measured by) $X$, the WSA is also influenced by another SCA measured by the variable $Y$ and characterized by the density function $f_{Y}(y)$, the utility functions $g_{a}(x, y)$ and $g_{b}(x, y)$ become many to one, and their specification becomes more difficult. Various shapes are possible for these functions, obvious examples being those obtained by revolving the one-dimensional functions in Figures 3 and 4 round the $g$-axis. The expected use for feature ' $a$ ' would be calculated as:

$$
E_{a}=\iint g_{a}(x, y) f_{x}(x) f_{Y}(y) d x d y
$$

assuming that SCA $X$ and $Y$ are statistically independent. The case where more than two SCAs have an impact on one WSA is treated similarly.

It is sometimes possible to transform the many-to-one problem to the framework of the one-to-one problem. This can be done by combining the applicable SCA distributions into one distribution, with the resultant variable having a one-to-one impact on the WSA in question, and for which a one-dimensional utility function can be specified as before. An example of this is the sight-line rotation rate, which 
is in itself a distribution that is completely determined by the target range and speed distributions.

For the one-to-many, or many-to-many case, where there is dependence between the various WSAs (for example missile accuracy), the situation becomes even more complex, as it is now not possible to evaluate the different WSAs in isolation. In such a case all the SCAs and WSAs that have an impact on the WSA in question must be taken into account simultaneously, and the distribution of the WSA in question must be calculated/simulated. This will, of course, depend on both the distributions characterizing the SCAs, as well as the assumed levels of the applicable WSAs. It is also more difficult to conceptualize a general utility function in this case, as the use of the feature is now also dependent on other WSAs. However, for a specific class of utility function such as discussed in the next paragraph, an interpretation similar to the one-to-one case can still be made.

\section{Specification of utility functions}

The expected use of a feature is directly influenced by the choice of utility function. As shown in Appendix A, the expression for the expected value in closed form becomes cumbersome for a pure ramp utility function, and would become considerably more so for a function such as in Figure 3. It was thus decided firstly to concentrate on the choice of a pure step utility function as in Figure 4, with the expected values as given in Appendix (A.9).

Another important motivation for this choice of utility function is that is allows for a second, more user-friendly interpretation of the expected utility of a WSA feature:

Given a downward step utility function, the expected use of the WSA attribute is the value of the (cumulative) distribution function of the SCA in the step point.

For example, if the WSA is 'Maximum Range', and the feature is ' $2000 \mathrm{~m}$ ', the utility function is a downward step function at 2000, and the expected use of the feature is numerically equal to the proportion of targets in the scenario that were at ranges less than $2000 \mathrm{~m}$. Conversely, for a WSA measured on a discrete scale such as 'Sight', with categories 'Telescope', 'Image Intensifier', and 'Thermal 
Imager' which links to the discrete SCA 'Visibility', with categories 'Day', 'Dusk', and 'Night', the utility function for the feature 'Image Intensifier' would have the value 1 for both 'Day' and 'Dusk', and 0 for 'Night'. This then again results in the value of the (discrete) cumulative distribution in the point 'Dusk' for the expected use of the feature.

This choice of utility function also allows more flexibility in the way that the questions can be phrased when gathering the SCA information, as discussed in the next section.

\section{APPLICATION OF CIM MODEL}

\section{Generation of scenarios and SCA distributions}

For the purpose of the ATM example, a scenario can be defined as a confrontation/situation where a number of missiles (and possibly other weapons) are used. The WSAs in question will dictate a number of SCAs for which information is needed to evaluate the use of the WSAs. The scale on which each SCA is measured must be clearly established, including the categories/cut-offs in the case of a discrete/continuous scale.

Given a scenario and the list of SCAs, the distributions must be quantified for each. For some (such as foliage density) it will be possible to obtain this by objective measurement. For others (such as target range) one will have to rely on the subjective collective opinion of a group of experts in the field, by using a structured information-gathering approach such as the Delphi technique.

It is important that the participants in the group should have both experience and authority, as well as the ability to think widely and futuristically. It is also important that the group be officially recognized as representative of the envisaged WS users.

Scenarios in which the proposed WS may be utilized must be generated. These will be futuristic, and can be grouped into the short, medium, and long term. These scenarios can more accurately be called 'battlefield scenarios', and should be at a 
level of detail which allows the characterisation of the required SCA distributions from the scenario description (see for example p42-5 in reference [3]. It should thus, for example, specify terrain down to the level where line-of-sight distances can be quantified, and weather conditions in such a way that temperatures and dust levels can be quantified. A possible format for a scenario could be a general section describing the setting in which the engagement takes place, followed by a more detailed section describing the blue and red forces involved more specifically.

The procedure for generating the scenarios can also vary. One possible approach is to invite experts on factors that would influence the application of the WS on a macro level, to give presentations to the group. These would be on present and projected future political, technological, economic and demographic trends, as well as on current and projected future strategies of own and enemy forces. Given this background, the group can formulate a set of likely battlefield scenarios, after which the distributions characterizing the SCAs can be quantified for each scenario.

It will be possible to quantify some of the SCA distributions largely independently of the WSAs under consideration (for example future target types). Others will be more awkward, such as the percentage of the battle that takes place at night - if the WS has a night-sight capability, blue forces will probably prefer (and hence force) night fighting, resulting in a significantly higher proportion of night fighting compared to a WS without this capability.

The phrasing of the questions to quantify the SCA distribution should be carefully planned. For example, for the SCA 'Visibility' with categories 'Day', 'Dusk', and 'Night' and entries $a \%, b \%$ and $c \%$, does this mean that

- $\quad a \%$ of the battle takes place by day, or

- $\quad a \%$ of the missiles that are fired, are fired by day?

Given the downward step utility functions as described earlier, the first interpretation is the required one. The expected use for the Image Intensifier 
option, for example, equals $(a+b) \%$, calculated from the SCA distribution:

\begin{tabular}{|c|c|c|}
\hline Day & Dusk & Night \\
\hline $\mathbf{a}$ & $\mathrm{b}$ & $\mathrm{c}$ \\
\hline
\end{tabular}

and utility function:

\begin{tabular}{|c|c|c|}
\hline Tel & II & TI \\
\hline 1 & 1 & 0 \\
\hline
\end{tabular}

The step utility function allows one to obtain directly the expected use of a WSA feature without formally specifying a utility function, and in this regard it is thus important to pay attention to the phrasing of questions. For example, instead of asking 'What \% of the scenario takes place in the categories Day, Dusk, and Night?' and then using the utility function as above to calculate the expected use of the II, one could also phrase the question as 'Given that the WS has the II feature, in what \% of the scenario will it be utilized?', and then directly obtaining the expected use of $(a+b) \%$. The two approaches provide the same final answers.

The model requires that all discrete SCA distributions be characterized by specifying the percentage per category. For the 'subjective' SCAs, these would be the consensus values obtained from the group. The continuous SCA distributions are modelled with a triangular distribution, for which three parameters must be specified:

- The mode is a user-friendly value, and is probably the value that people will tend to experience rather than the mean, especially in the case of highly skewed distributions.

- $\quad$ Either the absolute minimum and maximum values, or (say) the lower and upper 5th percentiles. Both have drawbacks. On the one hand, when supplying a minimum or maximum the respondent tends to over-estimate so as to make absolutely sure to cover the whole range. On the other hand, people's subjective interpretation and experience of a $5 \%$ value is different, and often differs little from the $10 \%$ value, for example. 


\section{Incorporating different development options}

Given that the expected and normalized use of the WS features have been calculated as in Table 4, these can be used to score the different development options. As indicated in Table 4, each option receives a score of between 0 and 1 , depending on the extent to which it has the various features, and a weighted sum of these scores is then calculated to yield a final performance score for each option. Note that this allows one to define the 'ideal' weapon system as one having all the features, whereupon the scores of the other options can be expressed as a percentage of this 'maximum attainable' score.

The choice of these WSA weights is once again problematic. One possible approach would be to try to correlate these with the ranking of the WS attributes identified using the AHP approach. Handel (Reference [4]) states that:

'... Material quality stands for the quality and performance of weapons which can be measured by their speed, range, fire-power, reliability, and durability. Many of these specifications can be measured, but the trade-offs between them cannot: the amount of emphasis to assign to reliability instead of to stat-of-the-art performance, the suitability of a certain weapon system to the quality of manpower available, and its performance in comparison with the enemy's weapons - all are very difficult to estimate, despite claims to the contrary'.

The important aspect, however, is that there are weights present. If they are not explicit, the decision-maker will use some invisible, subjective form of weighting between the WSAs to make a choice between the development options. The model serves to force these weights to become visible. A sensitivity analysis with respect to the choice of these weights should also be done. In Table 5 an example of the process applied to the ATM example is given, using a spreadsheet developed by $\mathrm{Mr} \mathrm{BL}$ Logan. 


\section{Incorporating different scenarios}

Finally, the above-mentioned process should be repeated for each scenario. In the final analysis, these scenarios can be weighted with respect to one another to determine a global performance figure for each of the development options. This bypasses the problem experienced in the AHP formulation of doing a direct comparison between the Infantry and the Paratroopers in order to determine the global weights, because it will be far easier for the user to assign weights to an 'impersonal' scenario on the basis of the scenario description. In fact, he can draw up a new scenario should he feel that his envisaged use of the WS is not adequately covered by the existing set of scenarios. In this way a database of application scenarios for the WS could be built up, which would also be useful for other planning purposes.

In this way then, the model supplies the values for the performance axis of the global choice reduction model discussed in Section 1.

\section{CONCLUSIONS}

We conclude the paper with a summary of the procedure for applying the CIM model, followed by a few general remarks on the current state of the model.

\section{Procedure for constructing the CIM}

The application of the model is done in a fairly sequential manner, and it is important that each step is properly executed before moving on to the next one.

1. From the Staff Requirement/User interviews/Crystal ball draw up a prioritized list of the functional requirements for the WS. Typical entries on the list are Man-portability, Maximum range, Terminal effect, Night-sight capability, etc.

2. Draw up a list of the physical attribute that the WS must have to be able to satisfy these, as well as the options available within each attribute from the manufacturer's point of view. (For example, for the sight capability, there are the options Tel, II of TI). This results in a list of WSAs and manufacturing options within each, which must be quantified in terms of the frequency/importance of use against the envisaged scenario in which the WS will be employed. The options will typically constitute discrete categories 
within each attribute, and it is important that these categories should be explicitly quantified.

3. For each WSA with its constituent options, determine exactly what information is required from the application scenario to quantify the use of the WS feature, and on what scale and in which format this information should be. This generates a list of SCAs.

4. Generate scenarios to such a level of detail that it is possible to quantify the use of the WS features within the scenario. These scenarios could be for different time horizons and environmental conditions, and should be the best guesses of a group of experts of the situations in which the WS is likely to be applied.

5. Conduct a Delphi-type exercise to obtain the information necessary to characterize the SCA distributions that cannot be quantified via 'objective' measurement.

6. Perform the calculations and/or simulations to calculate the expected use of the different WS features. This could be difficult, and would depend on the complexity of the WS and the nature of the interactions between the SCAs and the WS features. The interaction between the SCAs and the WSAs can be one of four types (one-on-one through many-on-many), depending on the information that is required from the application scenario and the WS itself to quantify the expected use of the options within the WSA.

7. Score the different development options in terms of the WS features, and calculate an overall score for each option. Do a sensitivity analysis on the weights for the overall score.

8. Calculate a global performance score for each development option over all the scenarios as a weighted total of the individual scenario performance scores. This supplies the performance value to be used in conjunction with the risk and cost scores in the global choice reduction model. 


\begin{tabular}{|c|c|c|c|c|c|c|c|c|c|c|c|c|}
\hline & \multicolumn{12}{|c|}{ WEAPON SYSTEM ATTRIBUTES } \\
\hline & \multicolumn{8}{|c|}{ Warhead Diameter $(\mathrm{mm})$} & \multicolumn{4}{|c|}{ Maximum Range $(\mathrm{m})$} \\
\hline & \multicolumn{4}{|c|}{ Single } & \multicolumn{4}{|c|}{ Tandem } & & & & \\
\hline & 105 & 115 & 127 & 150 & 105 & 115 & 127 & 150 & 1000 & 1500 & 2000 & 2500 \\
\hline $\begin{array}{l}\text { Percentage of Target } \\
\text { Population }\end{array}$ & $95 \%$ & $95 \%$ & $100 \%$ & $100 \%$ & $95 \%$ & $95 \%$ & $100 \%$ & $100 \%$ & $31,6 \%$ & $61,3 \%$ & $80,6 \%$ & $94,8 \%$ \\
\hline Weighting of WS Attributes & 1 & 1 & 1 & 1 & 1 & 1 & 1 & 1 & 1 & 1 & 1 & 1 \\
\hline Weighted Attribute Value & 0,95 & 0,95 & 1 & 1 & 0,95 & 0,95 & 1 & 1 & 0,316 & 0,613 & 0,806 & 0,948 \\
\hline Option 1 - Features & 0 & 0 & 0 & 0 & 0,95 & 0 & 0 & 0 & 0 & 0 & 0,806 & 0 \\
\hline Option 2 - Features & 0 & 0 & 1 & 0 & 0 & 0 & $\underline{0}$ & 0 & 0 & 0 & 0 & 0,948 \\
\hline Option 3 - Features & 0 & 0,95 & 0 & 0 & 0 & 0 & 0 & 0 & 0 & 0 & 0,806 & 0 \\
\hline Option 4 - Features & 0 & 0 & 0 & 0 & 0 & 0 & 1 & 0 & 0 & 0 & 0,806 & 0 \\
\hline Ultimate Weapon - Features & 0 & 0 & 0 & 0 & 0 & 0 & 0 & 1 & 0 & 0 & 0 & 0,948 \\
\hline
\end{tabular}

Table 5: Application c: model for ATM example 


\begin{tabular}{|l|c|c|c|c|c|c|c|c|}
\hline & \multicolumn{9}{|c|}{ WEAPON SYSTEM ATTRIBUTES } \\
\cline { 2 - 10 } & \multicolumn{1}{|c|}{ Minimum Range (m) } & \multicolumn{3}{|c|}{ Transportability } & \multicolumn{3}{c|}{ Accuracy } \\
\cline { 2 - 10 } & 50 & 150 & Manportable & Movable & $\begin{array}{c}\text { Vehicle } \\
\text { Only }\end{array}$ & Target 1 & Target 2 & Target 3 \\
\hline \hline $\begin{array}{l}\text { Percentage of Target } \\
\text { Population }\end{array}$ & $100 \%$ & $99,6 \%$ & $70 \%$ & $10 \%$ & $20 \%$ & $92,5 \%$ & $95,5 \%$ & $68,8 \%$ \\
\hline Weighting of WS Attributes & 1 & 1 & 1 & 1 & 1 & 1 & 1 & 1 \\
\hline Weighted Attribute Value & 1 & 0,996 & 0,7 & 0,1 & 0,2 & 0,925 & 0,955 & 0,688 \\
\hline Option 1 - Features & 0 & 0,996 & 0,7 & 0 & 0 & 0 & 0 & 0,688 \\
\hline Option 2 - Features & 0 & 0,996 & 0 & 0,1 & 0 & 0 & 0,955 & 0 \\
\hline Option 3 - Features & 0 & 0,996 & 0,7 & 0 & 0 & 0 & 0 & 0,688 \\
\hline Option 4 - Features & 1 & 0 & 0,7 & 0 & 0 & 0,925 & 0 & 0 \\
\hline \hline Ultimate Weapon - Features & 1 & 0 & 0,7 & 0,1 & 0,2 & 0 & 0,955 & 0 \\
\hline \hline
\end{tabular}




\begin{tabular}{|c|c|c|c|c|c|c|c|c|c|c|}
\hline & \multicolumn{10}{|c|}{ WEAPON SYSTEM ATTRIBUTES } \\
\hline & \multicolumn{3}{|c|}{ Sight Capability } & \multicolumn{7}{|c|}{ Reaction Time } \\
\hline & & & & \multicolumn{4}{|c|}{ Acquisition Time } & \multicolumn{3}{|c|}{ Flight Time } \\
\hline & $\begin{array}{l}\text { Telescope } \\
\text { (day) }\end{array}$ & $\begin{array}{c}\text { Image } \\
\text { Intensifier }\end{array}$ & TIS (24h) & $30 \mathrm{~s}$ & $15 s$ & $12 \mathrm{~s}$ & $10 \mathrm{~s}$ & $15 s$ & $12 \mathrm{~s}$ & $10 \mathrm{~s}$ \\
\hline $\begin{array}{l}\text { Percentage of Target } \\
\text { Population }\end{array}$ & $50 \%$ & $90 \%$ & $100 \%$ & $2,2 \%$ & $6,5 \%$ & $9 \%$ & $11,3 \%$ & $6,5 \%$ & $9 \%$ & $11,3 \%$ \\
\hline Weighting of WS Attributes & 1 & 1 & 1 & 1 & 1 & 1 & 1 & 1 & 1 & 1 \\
\hline Weighted Attribute Value & 0,5 & 0,9 & 1 & 0,022 & 0,065 & 0,09 & 0,113 & 0,065 & 0,09 & 0,113 \\
\hline Option 1 - Features & 0,5 & 0 & 0 & 0,022 & 0 & 0 & 0 & 0,065 & 0 & 0 \\
\hline Option 2 - Features & 0,5 & 0 & 0 & 0 & 0 & 0 & 0 & 0 & 0 & 0,113 \\
\hline Option 3 - Features & 0,5 & 0 & 0 & 0,022 & 0 & 0 & 0 & 0,065 & 0 & 0 \\
\hline Option 4 - Features & 0,5 & 0 & 0 & 0,022 & 0 & 0 & 0 & 0 & 0,09 & 0 \\
\hline Ultimate Weapon - Features & 0 & 0 & 1 & 0 & 0 & 0 & 0,113 & 0 & 0 & 0,113 \\
\hline
\end{tabular}




\begin{tabular}{|c|c|c|c|c|c|c|c|c|}
\hline & \multicolumn{6}{|c|}{ WEAPON SYSTEM ATTRIBUTES } & \multirow{3}{*}{$\begin{array}{l}\text { Figure of } \\
\text { Merit }\end{array}$} & \multirow{3}{*}{$\begin{array}{l}\text { Percentage } \\
\text { Ultimate } \\
\text { Weapon's } \\
\text { Performance }\end{array}$} \\
\hline & \multicolumn{3}{|c|}{ Sightline Rotation Rate (mrad/s) } & \multicolumn{3}{|c|}{ Firing Height Flexibility } & & \\
\hline & 16 & 26 & 33 & $\begin{array}{c}\text { lie } \\
\text { only }\end{array}$ & $\begin{array}{c}\text { kneel } \\
\text { only }\end{array}$ & $\begin{array}{l}\text { stand } \\
\text { only }\end{array}$ & & \\
\hline $\begin{array}{l}\text { Percentage of Target } \\
\text { Population }\end{array}$ & $97,9 \%$ & $99,2 \%$ & $99,6 \%$ & $30 \%$ & $30 \%$ & $40 \%$ & & \\
\hline Weighting of WS Attributes & 1 & 1 & 1 & 1 & 1 & 1 & & \\
\hline Weighted Attribute Value & 0,979 & 0,992 & 0,996 & 0,3 & 0,3 & 0,4 & & \\
\hline Option 1 - Features & 0 & 0,992 & 0 & 0,3 & 0 & 0 & 6,02 & $74,1 \%$ \\
\hline Option 2 - Features & 0,979 & 0 & 0 & 0 & 0,3 & 0,4 & 6,29 & $77,4 \%$ \\
\hline Option 3 - Features & 0 & 0,992 & 0 & 0,3 & 0 & 0 & 6,02 & $74,1 \%$ \\
\hline Option 4 - Features & 0 & 0 & 0,996 & 0,3 & 0,3 & 0,4 & 7,04 & $86,6 \%$ \\
\hline Ultimate Weapon - Features & 0 & 0 & 0,996 & 0,3 & 0,3 & 0,4 & 8,13 & $100 \%$ \\
\hline
\end{tabular}




\section{Summary}

Having applied the model described above to the problem of reducing the number of options during the concept phase of the development of an ATM, we feel that the basic methodology and feasibility of the method has been demonstrated. Some more specific remarks are:

- The model is not yet complete in terms of the quantification of some of the CIM interactions, which still need some further refinement.

- The cost and technical risk axes must be incorporated.

- It provides a common basis for discussion between the user and the supplier, in quantifying the interaction between weapon system attributes and the intended operational environment.

- The process quantifies the user's operational requirement, and the 'shape' of the weapon needed to satisfy these requirements.

- It is convenient to rate the different options relative to an 'ideal' system.

- Applying the model has clearly indicated where our input data is not complete.

In conclusion, we feel that the model has provided a useful map between the weapon system and the intended application scenario. This map can serve as a common, quantified basis for discussion between the parties involved, and can also clarify marketing strategies.

\section{REFERENCES}

[1] Saaty, T.L., The Analytical Hierarchy Process, McGraw-Hill, 1980.

[2] Courtright, J.F. and Kidd, S.F., Scenario Analysis: A Tool for Planning $O T \& E$, Proceedings of the Human Factors Society - 30th Annual Meeting, 1986.

[3] US Army Engineering Design Handbook: Army Weapon System Analysis, Part 2, DARCOM PAMPHLET 706-102, October 1979.

[4] Handel, M., Numbers Do Count: The Question of Quality Versus Quantity, Journal of Strategic Studies, Vol 4 no 3, p225-260, 1981.

[5] Expert Choice program and user manual, by Decision Support Software Inc., McLean, Virginia, 1983,84,85. 


\section{APPENDIX A}

\section{CALCULATION OF THE EXPECTED USE OF A WSA AND RELATED QUANTITIES}

Assume that the variable $X$ has a triangular distribution, i.e.

$$
\begin{aligned}
f_{x}(x) & =a\left(x-x_{0}\right) & & x_{0} \leq x<x_{1} \\
& =b\left(x_{2}-x\right) & & x_{1} \leq x \leq x_{2} \\
& =0 & & \text { elsewhere }
\end{aligned}
$$

where $x_{0}=$ minimum value, $x_{1}=$ mode, and $x_{2}=$ maximum value of $X$, and $a>$ $0, b>0$ the slope parameters. Subject to the restriction that $f_{X}$ is a density function, the values of $a$ and $b$ can be determined as

$$
a=\frac{2}{\left(x_{2}-x_{0}\right)\left(x_{1}-x_{0}\right)}, \quad b=\frac{2}{\left(x_{2}-x_{0}\right)\left(x_{2}-x_{1}\right)}
$$

Consider now a utility function $g(x)$ which is an upward or downward step or ramp,
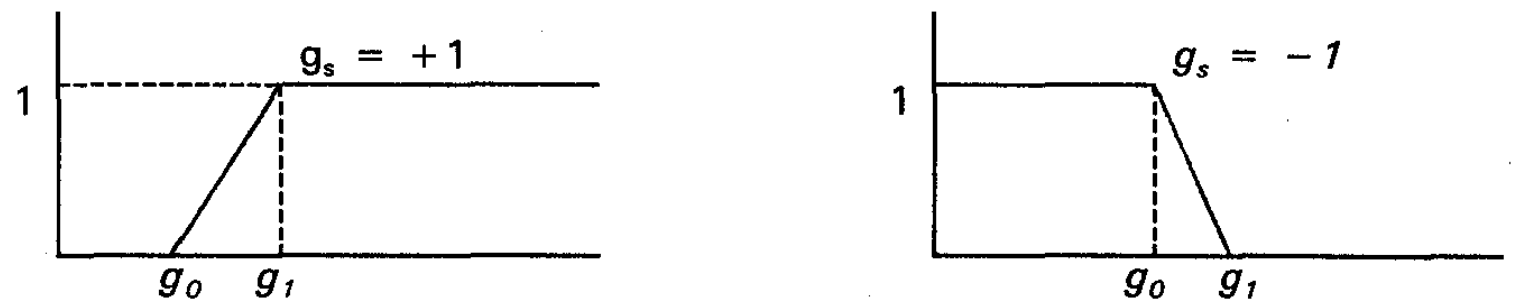

with parameters $g_{s}= \pm 1$ and $g_{0} \leq g_{1}$. Note that the function reduces to a step function if $g_{o}=g_{1}$.

The four possible shapes of $g(x)$ can be combined as in the following definition:

$$
\begin{array}{lr}
g(x)=\left(1-g_{s}\right) / 2 & x \leq g_{0} \\
g(x)=/\left(g_{0} \neq g_{1}\right) g_{s} \frac{\left(x-g_{0}\right)}{\left(g_{1}-g_{0}\right)} & g_{o}<x \leq g_{1} \\
g(x)=\left(1+g_{s}\right) / 2 & g_{1}<x
\end{array}
$$

where $I(A)$ is the indicator function, returning the value 1 if $A$ is true, and 0 otherwise. 
Our first requirement is to derive a closed-form expression for the expected value of $g(X)$,

$$
E[g(X)]=\int_{x_{0}}^{x_{2}} g(x) f_{X}(x) d x
$$

that can readily be incorporated in, for example, a spreadsheet. To calculate $E[g(X)]$, account must be taken of the sequence in which $x_{0}, x_{1}, x_{2}$ and $g_{0} g_{1}$ can occur. There are ten distinct possibilities, ranging from $x_{0}<x_{1}<x_{2}<g_{0}<g_{1}$ to $g_{0}<g_{1}<x_{0}<x_{1}<x_{2}$, of which $x_{0}<g_{0}<x_{1}<g_{1}<x_{2}$ is the most likely from a practical point of view.

Considering this last case by way of example, we find that (A.4) evaluates to:

$$
\begin{aligned}
E[g(X)] & =\frac{a\left(1-g_{s}\right)}{4}\left(g_{0}-x_{0}\right)^{2} \\
& +\frac{a g_{s}}{\left(g_{1}-g_{0}\right)}\left[\frac{\left(x_{1}^{3}-g_{1}^{3}\right)}{3}-\frac{\left(x_{0}+g_{0}\right)}{2}\left(x_{1}^{2}-g_{0}^{2}\right)+x_{0} g_{0}\left(x_{1}-g_{0}\right)\right] \\
& -\frac{b g_{s}}{\left(g_{1}-g_{0}\right)}\left[\frac{\left(g_{1}^{3}-x_{1}^{3}\right)}{3}-\frac{\left(x_{2}+g_{0}\right)}{2}\left(g_{1}^{2}-x_{1}^{2}\right)+x_{2} g_{0}\left(g_{1}-x_{1}\right)\right] \\
& +\frac{b\left(1+g_{s}\right)}{4}\left(g_{1}-x_{2}\right)^{2}
\end{aligned}
$$

As can been seen, these expressions are somewhat tedious to evaluate, and the answer would depend on a 10-way switch (according to the different permutations of the parameters) that will all have to be incorporated in the spreadsheet.

We first consider the pure upward or downward step function with $g_{0}=g_{1}=g_{b^{\prime}}$ that is

$$
\begin{array}{ll}
g(x)=\left(1-g_{s}\right) / 2 & x \leq g_{b} \\
g(x)=\left(1+g_{s}\right) / 2 & x>g_{b}
\end{array}
$$

with $g_{s}= \pm 1$. 
Four permutations for $x_{0}, x_{1}, x_{2}$, and $g_{b}$ must be considered:

1) $x_{0}<x_{1}<x_{2}<g_{b}$, for which $E[g(X)]=0$ if $g_{s}=+1$, and $=1$ if $g_{s}=-1$

2) $x_{0}<x_{1}<g_{b}<x_{2}$

3) $x_{0}<g_{b}<x_{1}<x_{2}$

4) $g_{b}<x_{0}<x_{1}<x_{2}$, for which $E[g(X)]=1$ if $g_{s}=+1$, and $=0$ if $g_{s}=-1$

For case 2) we find:

$$
\begin{aligned}
E[g(X)] & =\int_{x_{0}}^{x_{1}} \frac{\left(1-g_{s}\right)}{2} a\left(x-x_{0}\right)+\int_{x_{1}}^{g_{b}} \frac{\left(1-g_{s}\right)}{2} b\left(x_{2}-x\right)+\int_{g_{b}}^{x_{2}} \frac{\left(1+g_{s}\right)}{2} b\left(x-x_{2}\right) \\
& =\frac{\left(1-g_{s}\right)}{4}\left\{a\left(x_{1}-x_{0}\right)^{2}+b\left(x_{2}-x_{1}\right)^{2}\right\}+\frac{b g_{s}}{2}\left(x_{2}-g_{b}\right)^{2}
\end{aligned}
$$

For case 3) we find similarly:

$$
E[g(X)]=-\frac{a g_{s}}{2}\left(g_{b}-x_{0}\right)^{2}+\frac{\left(1+g_{s}\right)}{4}\left\{a\left(x_{1}-x_{0}\right)^{2}+b\left(x_{2}-x_{1}\right)^{2}\right\}
$$

Utilizing (A.2), it can readily be shown that the expression in braces in (A.7) and (A.8) equals 2.

Combining all the results, we find that for $X$ triangularly distributed as in $(A .1)$, with a step utility function $g$ as in (A.6),

$$
\begin{aligned}
& E[g(X)]=\int g(x) f_{X}(x) d x \\
& =\left(1+g_{s}\right) / 2 \quad g_{b}<x_{0} \\
& =\left[1+g_{s}-a g_{s}\left(g_{b}-x_{0}\right)^{2}\right] / 2 \quad x_{0} \leq g_{b}<x_{1} \\
& =\quad\left[1-g_{s}+b g_{s}\left(x_{2}-g_{b}\right)^{2}\right] / 2 \quad x_{1} \leq g_{b}<x_{2} \\
& =\left(1-g_{s}\right) / 2 \quad x_{2}<g_{b}
\end{aligned}
$$

with

$$
a=\frac{2}{\left(x_{2}-x_{0}\right)\left(x_{1}-x_{0}\right)}, \quad b=\frac{2}{\left(x_{2}-x_{0}\right)\left(x_{2}-x_{1}\right)}
$$


Secondly, we consider the calculation of the parameters of the triangular distribution (A.1), given estimates of two percentiles and either the mean or the mode. The situation is depicted graphically in the following figure.

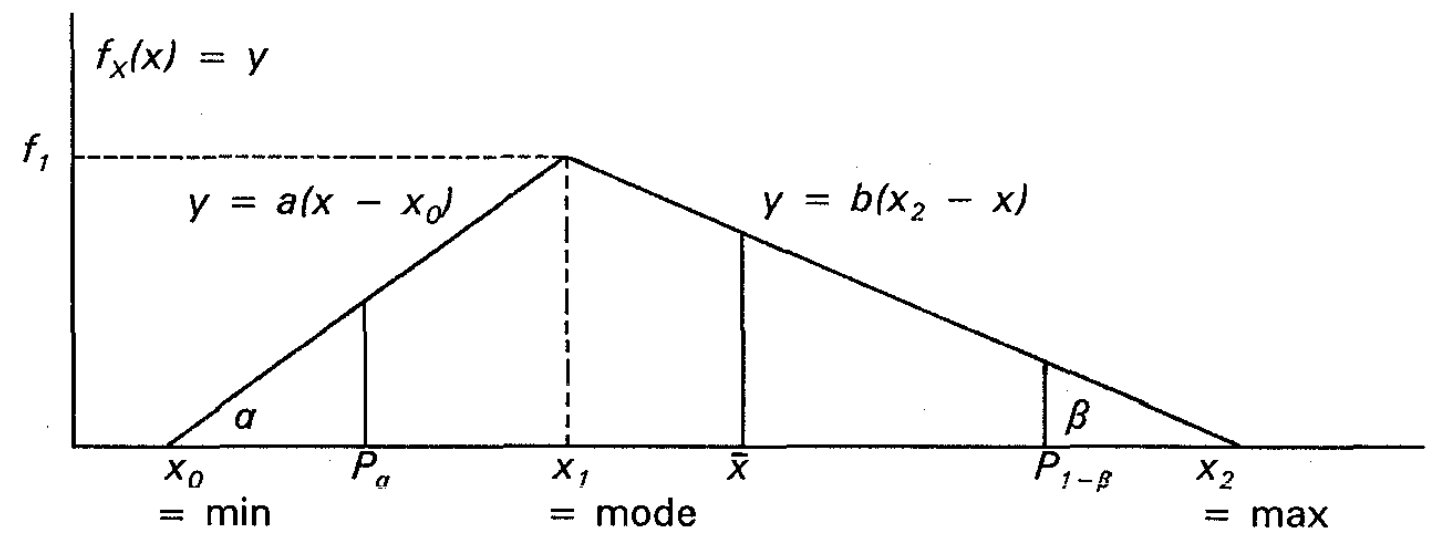

The problem is simply to determine the values of $x_{0} x_{1}$ and $x_{2}$, given $a, P_{a}, \beta, P_{1-\beta}$ and either $\bar{x}$ or $x_{1}$.

From the definition of the percentiles $P_{\alpha^{\prime}} P_{1-\beta}$, and the requirement that $f_{X}$ be a density, the following equations can be shown to hold:

$$
\begin{aligned}
& a\left(x_{1}-x_{\sigma}\right)\left(x_{2}-x_{\sigma}\right)=\left(P_{a}-x_{\sigma}\right)^{2} \\
& \beta\left(x_{2}-x_{1}\right)\left(x_{2}-x_{0}\right)=\left(x_{2}-P_{1-\beta}\right)^{2}
\end{aligned}
$$

Assuming first that $\bar{x}$ is given, and $x_{1}$ is unknown, the following mixed quadratic in $x_{0}$ and $x_{2}$ follows after some manipulation of the above relations:

$$
\beta(\alpha-1) x_{0}^{2}+2 \beta\left(P_{a}-a x_{2}\right) x_{0}+\beta\left(a x_{2}-P_{a}\right)^{2}-a\left(x_{2}-P_{1-\beta}\right)^{2}=0
$$

The definition of $\bar{x}$ provides a second relationship between $x_{0}$ and $x_{2}$, as

$$
\bar{x}=(a+b) x_{1}^{3} / 3-\left(a x_{0}+b x_{2}\right) x_{1}^{2} / 2+\left(a x_{0}^{3}+b x_{2}{ }^{3}\right) / 6
$$

To solve the last two equations for the unknown variables is awkward. One could iterate for $x_{2}$, starting from the given $P_{1-\beta}$, and calculate $x_{0}$ from (A.12), after which $x$, can be calculated from (A.13).

Alteratively, we assume that $x_{1}$ is known. This is in line with the decision that 
from a practical point of view the scenario attribute distributions will be characterised by their lower and upper $5 \%$ percentiles and the most likely value (the mode).

Assuming that $x_{1}=$ mode is known simplifies matters considerably. From (A.10) and (A.11) with $x_{1}=m$ we have:

$$
\begin{aligned}
& x_{2}=x_{0}+\frac{\left(P_{\alpha}-x_{0}\right)^{2}}{\alpha\left(m-x_{0}\right)} \\
& x_{0}=x_{2}-\frac{\left(x_{2}-P_{1-\beta}\right)^{2}}{\beta\left(x_{2}-m\right)}
\end{aligned}
$$

Combining these two equations yields a 4th-order equation in the remaining variable, which can be solved in a closed form. In practical applications we employ a graphical procedure which simply superimposes two plots on top of one another. The first one is of $O \leq x_{o} \leq P_{a}$ vs $x_{2}$ calculated from (A.14), and the second that from the first step vs $x_{0}$ calculated from (A.15). These two graphs intersect in more than one point. (The second equation is not a function). The maximum value of $x_{2}$ on the vertical axis represents the solution.

Having obtained $x_{0}$ and $x_{2}$, and given $m=x_{1}$, the remaining parameters $a$ and $b$ can readily be calculated from (A.9).

Finally, for simulation purposes we need the inverse triangular distribution in closed form. The distribution is:

$$
\begin{aligned}
F(x) & =a\left(x-x_{0}\right)^{2} / 2 & & x_{0} \leq x \leq x_{1} \\
& =a\left(x_{1}-x_{0}\right)^{2} / 2 & & x=x_{1} \\
& =F\left(x_{1}\right)+b\left(x-x_{1}\right)\left(2 x_{2}-x-x_{1}\right) / 2 & & x_{1}<x \leq x_{2}
\end{aligned}
$$

The inverse distribution is:

$$
\begin{array}{ll}
F^{-1}(u)=x_{0}+\sqrt{2 u / a} & x_{0} \leq u<x_{1} \\
F^{-1}(u)=x_{2}-\sqrt{\left(x_{2}-x_{1}\right)^{2}+2\left[F\left(x_{1}\right)-u\right] / b} & F\left(x_{1}\right) \leq u \leq 1
\end{array}
$$

